\title{
Growing rod concepts: state of the art
}

\author{
Muharrem Yazici $\cdot$ Z. Deniz Olgun
}

Received: 7 February 2012/Revised: 10 April 2012/Accepted: 15 April 2012/Published online: 8 May 2012

(C) Springer-Verlag 2012

\begin{abstract}
Introduction Early-onset spinal deformities present multiple challenges to the surgeon. They may be rapidly progressive and unresponsive to conservative treatment, necessitating surgical intervention at an early age.

Materials and methods This text attempts to provide a review of current literature and to summarize the authors' opinions.

Results This paper attempts to concisely review available literature regarding the growing rod's inception, evolution, technique, results, and complications and answers some of the controversy still surrounding it.

Conclusions The growing rod is one of the first, most evolved, most popular and one of the most heatedly discussed technique of fusionless spinal instrumentation.
\end{abstract}

Keywords Early-onset scoliosis · Surgery ·

Growth preservation

\section{Introduction}

Due to its in many ways central location in the body, the vertebral column plays a huge role both physiologically and functionally in the growth and development of the child's body. Any interference with the normal growth and development of the spinal column, be it deformity or even the treatment thereof, will invariably have consequences for the entire body. This is one of the many reasons why early-onset spinal deformities in children have become a

M. Yazici $(\bowtie) \cdot$ Z. D. Olgun

Department of Orthopaedics and Traumatology,

Hacettepe University, Sihhiye, Ankara 60100, Turkey

e-mail: yazioglu@hacettepe.edu.tr heatedly discussed subject in spinal surgery in the past few years. Advances in implant technology and new understanding of disease processes have led to groundbreaking changes in the treatment of adult and adolescent deformities, some of which have been transferred to early-onset disorders as well. However, the large potential of growth in this special patient population presents a wide spectrum of dilemmas and complications in their treatment.

Spinal deformities that cannot be controlled by conservative means require surgical intervention. This statement is no less true for early-onset spinal disorders. While no concise blanket treatment, conservative or surgical, exists for the management of the myriad conditions that make up early-onset spinal deformities, the growing rod is one of the most popular and widely used methods.

\section{Conception and history}

One of the most powerful tools against progressive deformity in the armament of the spinal surgeon is fusion. While one of the most intricately constructed and in health stable structures of the skeletal system, the dynamic relationship between vertebrae becomes the very reason why deformities after a certain magnitude progress. Fusion abolishes this dynamic relationship, turning the spine into a rigid construct, aiming to establish balance by decreasing or correcting deformity. In an adult or an adolescent with very little growth remaining, the loss of this mobility is usually well compensated. In the young child, however, it causes unique problems, and correction of the deformity is but a lesser component of it. More essential is the struggle to preserve the growth potential of the spine.

Due to its central, or axial, location in the body, the spine is in the center of all growth and development taking 
place around it. Its growth, or lack thereof, affects the thorax, the abdomen, and the pelvis. Perhaps the most significant relationship, however, is with the thorax and its contents: the lungs.

The growth of these two structures proceeds together after birth, continuing in a non-linear fashion. Rapid acceleration of growth occurs before the age of three and during the pubertal growth spurt [1]. In a child with a healthy spine, the volumes of both these structures are proportional to height. Lung growth is a complex process, with different parts of the lungs growing at different rates and different times during the life of the child, and of these, alveolar development continues well into the teens. Disruption of growth as occurs in early-onset spinal deformity leads to many pulmonary consequences, one of which has been defined as the thoracic insufficiency syndrome, the inability of the thorax to support normal lung function [2].

Growth of the deformed spine, whether the deformity be of congenital or idiopathic in origin, is not normal. This may be due to deficient or excessive growth centers as found in congenital deformities, or asymmetric compressive and distractive forces exerted upon growth plates in all, or inherent, yet-to-be-discovered faults within the growth plate itself. It is, however, well known that a fused spine does not grow. It has been previously published that both vital capacity and lung diffusion capacity are negatively affected by early fusion [3]. While the adage of 'a short but straight spine is better than a long and crooked one' has been an undisputed motto for spinal surgery in the past, these recent developments regarding complications after the loss of growth have spurred the research for methods to achieve a long and straight spine.

Once the drawbacks of early fusion became apparent, the search for methods to postpone final fusion for as long as possible while maintaining an amount of control over the deformity began. These so-called delaying tactics are conservative methods that are combined with close observation of the patient [4]. As it is now known that the greater the age at commencement of growing rod treatment and therefore the fewer the surgeries the child has to undergo, the less chance of complications occurring exists; these delaying tactics are used for growing rod treatment as well. Among these are brace treatment, serial casting and halogravity traction. Bracing, one of the oldest conservative methods for the control of spinal deformity, can be used in patients with idiopathic or idiopathic-like curves if the compressive action of the brace does not interfere with respiratory function, as may occur in patients with neuromuscular disorders and other medical conditions. In some cases, serial casting may be preferable over a brace as it is basically a brace that cannot be removed and therefore negates cooperation problems. Casting in spinal deformity attempts to correct the deformity by an indirect molding force to the rib hump and some amount of traction with a neck piece. It is repeated every 2-3 months over a period of 6-9 months and often successful in moderate deformities. Both brace and cast treatment are associated with skin irritation and pressure sores, respiratory difficulty and rib deformations.

If fusion is out of the picture and delaying tactics no longer work, instrumentation becomes essential for the control of deformity. The first instrumentation to gain popular application, the Harrington rod, was also the first implant to be used for fusionless surgery. Reports of Harrington rods used without fusion go back as early as the late 1970s and early 1980s [5]. Moe et al. [6] described a technique similar to that used today, where the subcutaneous insertion of a Harrington rod was intended to function like an internal brace, and it was supplemented with the use of external orthosis postoperatively. No segments were fused and the rod distracted periodically according to the indication of an increase in deformity by $10^{\circ}$. Patients in this series attained $84 \%$ of expected growth in the instrumented segment, although many required unplanned surgery due to implant-related complications. This high complication rate caused reports of a discouraging nature [7], including Mineiro and Weinstein [8], who questioned the method due to this and less-than-expected growth in the instrumented segment. In their 2002 report, Acaroglu et al. published the results of 12 patients, where a significant increase was observed in rotational deformity, although coronal plane deformity could be controlled with subcutaneous rod insertion without fusion. According to the authors, this situation may have two explanations: one, the subcutaneous rod is not able to control rotational plane deformity as effectively as that in the coronal plane and two, the spontaneous fusion or ankylosis that is thought to take place in the uninstrumented region of the spine accounting for the increase in rigidity at definitive fusion may have caused an increase in rotation with a mechanism similar to the crankshaft phenomenon [9].

These and other reports caused a setback in the popularity of the method during the 1990s and early 2000s, until the 2005 report by Akbarnia et al. where the technique was revised to include two instead of one rod on the concave side, subperiosteal dissection only at anchor sites to minimize the possibility of implant failure and periodic lengthening twice a year regardless of the progression of the curve. They achieved good correction and control of deformity and an average T1-S1 length increase of $1.21 \mathrm{~cm} /$ year. While $48 \%$ of the patients had complications of any nature, only $17 \%$ required unplanned surgery. The authors concluded that the dual growing rod technique was safe and effective, and had an acceptable rate of complications compared with previous reports while preserving near-normal growth [10]. Consistently better results 
have been reported with the use of double rods instead of a single one [11, 12].

Changes in implant technology have led to changes in anchor selection as well. The technique, originally defined for Harrington instrumentation, has over time evolved to include hooks, bisegmental claw formations using hooks, specifically designed growing (or tandem) connectors and the use of cantilever torsion maneuvers for deformity correction alongside the original primary distraction forces.

\section{Technique}

Selection of instrumentation levels is performed by the analysis of deformity in standing anteroposterior, lateral and especially traction X-rays taken under anesthesia. As distraction is still the primary method for deformity correction, Harrington principles are used and vertebrae within the stable zone are selected as distal and proximal anchor points (Figs. 1, 2).
The approach is begun with a midline longitudinal incision. Depending on the preference of the surgeon, two separate incisions or one long incision can be used. Submuscular dissection is continued through the incision but only single motion segments at the anchor points are exposed subperiosteally. The implants of choice are placed at the previously selected anchor levels. Either hooks, claw-constructs using hooks or pedicle screws can be used as implants; the author prefers pedicle screws at both ends as previous studies have reported increased stability with their use and transverse connectors [13]. Only the facet joints at the anchor levels are excised and fusion performed; inter- and intraspinous ligaments and facet joint capsules at adjacent levels are preserved. Once implant placement is complete, four rods, two proximal and two distal, are contoured according to the sagittal alignment of the spine, taking into account natural kyphosis. The rods are placed in a submuscular location within grooves established in the paravertebral muscles and engaged into the anchor implants. A transverse connector can be used
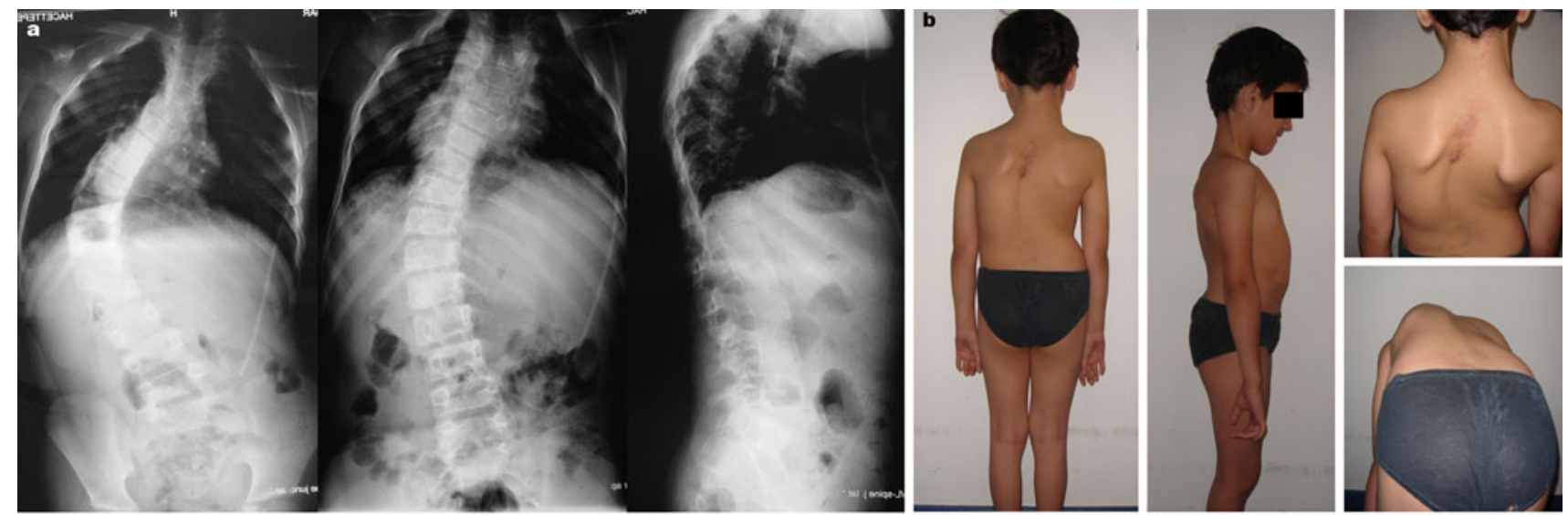

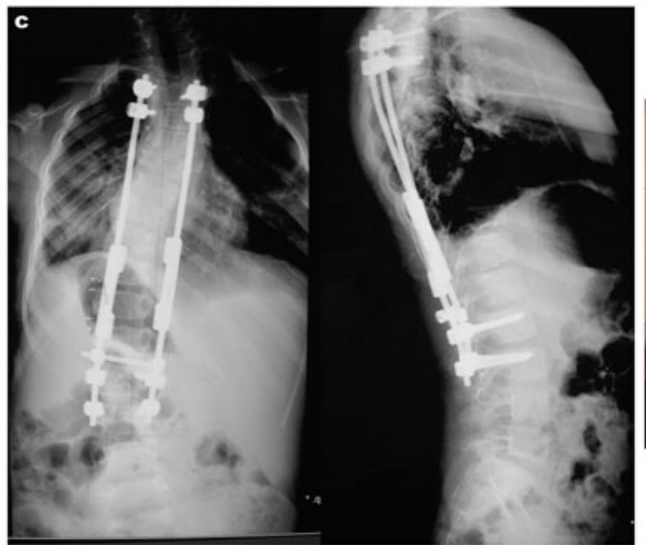

Fig. 1 The patient in this figure is a 105-month-old girl with a diagnosis of congenital scoliosis. She underwent growing rod surgery at a standing height of $135 \mathrm{~cm} 1$ year after the excision of a diastometamyelic spur and dural repair, and a ventriculoabdominal shunt implantation in the neonatal period. a Standing anterior-

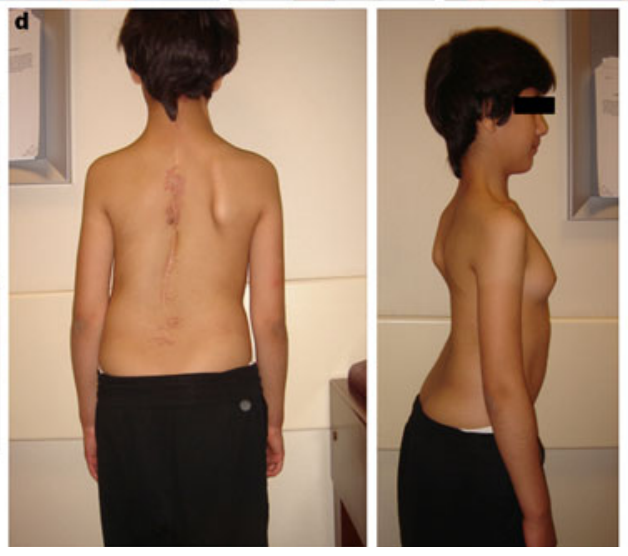

posterior, supine traction under general anesthesia, and lateral standing radiographs at index surgery. b Preoperative clinical appearance of the patient. c Standing anterior-posterior and lateral X-rays after index surgery. d Patient's clinical appearance before her fifth lengthening 

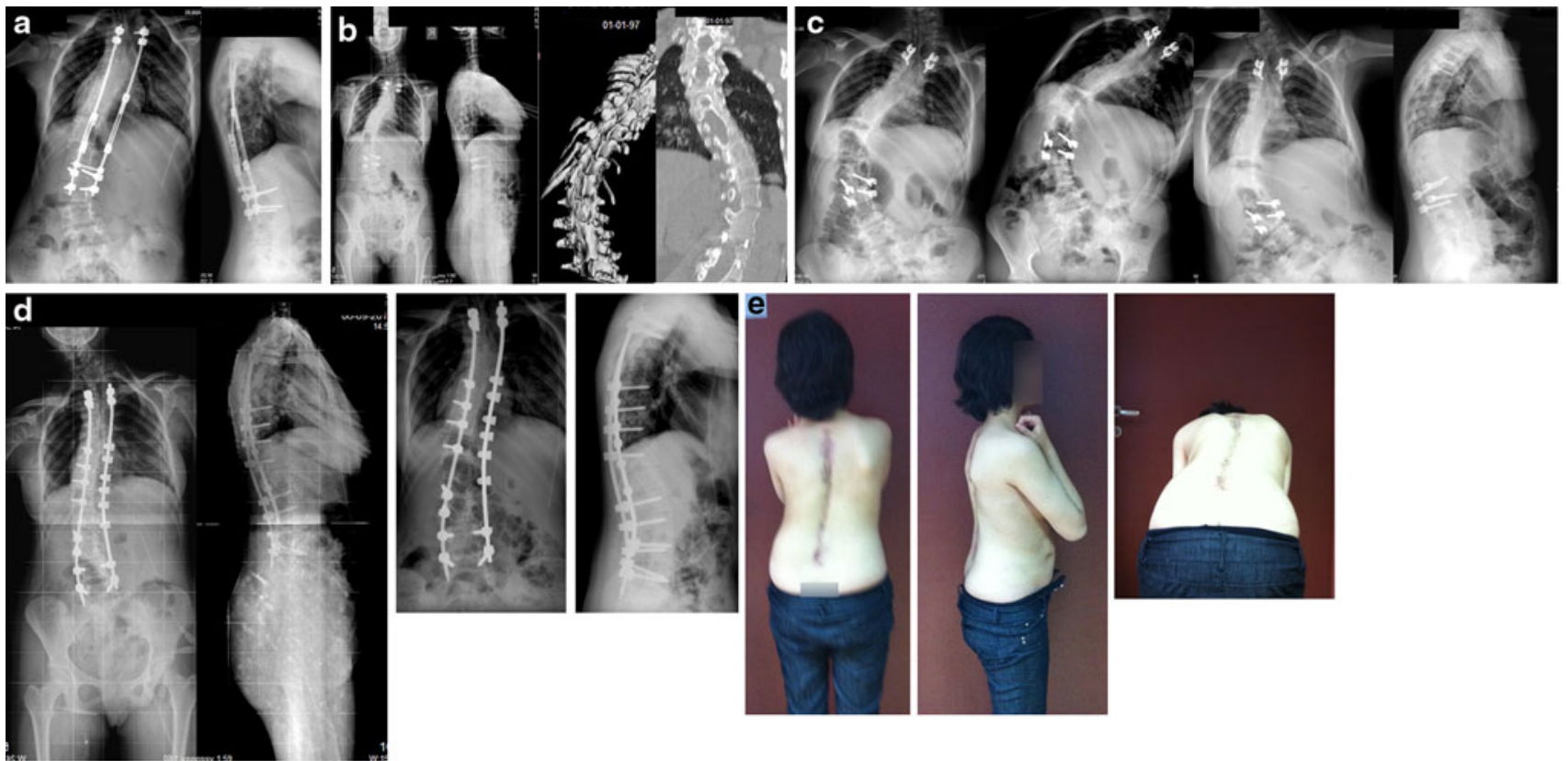

Fig. 2 The patient from Fig. 1 underwent a total of 11 lengthenings. No complications requiring unplanned surgery were encountered. Her standing height before finalization of growing rod treatment (implant removal) was $153.5 \mathrm{~cm}$, her forced vital capacity $1.90 \mathrm{~L}(70 \%$ of expected) and forced expiratory volume in the first second was $91 \%$ of expected. a Standing anterior-posterior and lateral views of the patient before finalization of growing rod treatment. b Patient's standing anterior-posterior and lateral X-rays after removal of implants. The CT sections show the extent of her congenital deformity

both proximally and distally to connect the two rods together. A growing connector (or tandem connector) is placed between the two rods and using cantilever torsion, the proximal and distal rods are connected to each other. Distraction and compression between segments are used to achieve better spinal balance. Closure proceeds in an anatomic fashion, with or without the placement of a drain left to the surgeon's discretion.

An external TLSO brace is used for the first 6 months following index surgery. Regardless of the evolution of the deformity, the rods are distracted every 6 months with preplanned lengthening surgery. No external support is used after lengthening sessions, which are usually performed on an outpatient basis.

Several additional interventions have been described for growing rod treatment including apical fusion to arrest the increase in vertebral rotation and the crankshaft phenomenon, annulotomies and other methods of spinal release. Caubet et al. [14] reported on the results of halo-gravity traction compared with surgical release before the implantation of fusionless, expandable spinal devices. They observed that spinal release resulted in an improved correction of scoliosis over halo-gravity traction or no release, but halo-gravity traction was superior in the correction of kyphosis while thoracic spine height increased

at this stage. c Standing anterior-posterior, right-side bending, leftside bending and lateral X-rays of the patient 6 months after implant removal. Obvious increase in deformity and loss of trunk balance can be appreciated. Patient underwent definitive treatment with posterior instrumentation and fusion after this follow-up appointment. d Standing anterior-posterior and lateral radiographs following definitive fusion. The deformity has been reasonably corrected and balance reestablished. e Patient's clinical appearance 2 months after definitive fusion

initially more in the halo group, but was surpassed by the spinal release group at follow-up. A high rate of device complications was seen in the spinal release group, and neurologic complications ( $8 \%$ total) were more frequent in the halo-gravity group.

\section{Results}

The first extensive report of the modern dual growing rod technique was the aforementioned 2005 study by Akbarnia et al. In this study, the authors followed 23 patients operated on with the dual growing rod technique and included only patients with follow-up of at least 2 years after index surgery, seven of whom underwent final fusion during the duration of the study. The patients had an average of 6.6 lengthenings. Mean scoliosis improved from $82^{\circ}$ to $36^{\circ}$ at final follow-up while T1-S1 length increased from an average of 23.01 to $28.00 \mathrm{~cm}$, averaging a length increase of $1.21 \mathrm{~cm} /$ year. They also reported an improved space available for the lung ratio in their patients, going from 0.87 to 1 at the conclusion of the study. The authors concluded that the dual growing rod technique maintains the correction achieved at index surgery while allowing growth to carry on. 
Akbarnia et al. followed this report with a 2008 study of 13 patients who all underwent final fusion and had a minimum of 2-year follow-up. None of the patients had undergone previous surgery and all had non-congenital curve etiologies. Scoliosis correction was similar with the previous report, going from $81^{\circ}$ to $27.7^{\circ}$ after final fusion while T1-S1 length increased from 24.4 to $29.3 \mathrm{~cm}$, averaging $1.46 \mathrm{~cm} /$ year. The authors also compared patients undergoing lengthening procedures at most every 6 months with those where the period between lengthenings was longer and determined that more frequent lengthenings resulted in increased rates of growth (1.8 vs. $1.0 \mathrm{~cm} /$ year) and better control of deformity (scoliosis correction 79 vs. $48 \%$ ). In a 2010 study by Farooq et al. [15], the results of a series of 88 patients treated with a single growing rod and lengthening performed at an average of 9-month intervals, scoliosis was observed to have improved from $73^{\circ}$ to $44^{\circ}$ with an annual growth rate of $1.04 \mathrm{~cm} /$ year.

Several factors regarding the nature of the child's deformity are thought to affect outcome. The effect of thoracic kyphosis in growing rod treatment has been investigated in a study by Schroerlucke et al. [16]. The authors compared complication rates in patients with normal kyphosis and abnormal thoracic kyphosis. In the 90 patients they analyzed, the authors reported that patients were with hyperkyphosis were 3.1 times more likely to experience implant-related complications than patients with normal kyphosis, and patients with hypo or hyperkyphosis were more likely to suffer from general medical complications as well. The authors suggested close monitorization of such patients and family counseling. These findings should be taken into account when surgical strategy is planned and any measure taken to improve fixation in hyperkyphotic patients. In order to avoid junctional kyphosis at the proximal anchor sites, the rods should be contoured into kyphosis at the top of the construct while interspinal ligaments should be kept intact. The anchor sites can be extended to $\mathrm{T} 2$ or even higher for this purpose.

Although growing rod treatment was conceived for idiopathic and idiopathic-like deformities, its use in other indications has become widespread mainly due to the highly progressive nature of non-idiopathic deformities. In a 2010 paper by Elsebai et al., the results of 19 patients with congenital scoliosis undergoing growing rod treatment were retrospectively studied. The patients underwent index surgery at an average of 6.9 years and had a minimum of 2 years of follow-up, at the conclusion of which their scoliosis improved from an average of $66^{\circ}$ to $47^{\circ}$ while T1-S1 length increased from a mean of 268.3 to $315.4 \mathrm{~mm}$, showing an annual growth rate of $11.7 \mathrm{~mm} /$ year. Their space available for the lung ratio also improved from 0.81 to 0.94 at last follow-up. While these numbers are lower than those reported for idiopathic and idiopathic-like series, they are close, and while controversy still exists, making growing rod treatment a safe and effective treatment technique in congenital scoliosis [17]. Similarly favorable results have been achieved with the use of growing rods for the treatment of spinal muscular atrophy (SMA) as well [18, 19].

The effect of growing rod treatment on the rib cage has been studied. Sabourin et al. [20] used low-dose stereoradiographic imaging (EOS) to analyze the changes in thoracic geometry following the application of a growing rod. The authors used a single-rod construct in the study and reported that with $\mathrm{Cobb}$ correction from $50.8^{\circ}$ to $26^{\circ}$, a complex three-dimensional effect took place on the rib cage, improving its structure in both the coronal and sagittal planes, although this was less extensive than in the case of a spinal arthrodesis. The authors conclude that longer follow-up and more patients should be analyzed in order to improve understanding of correction forces exerted upon the rib cage.

\section{Complications}

Although the logic behind its technique is sound and innovative, a high complication rate reported in the first series regarding its use has caused the growing rod to be met with skepticism and prejudice in the 1990s and early 2000s. Among the most common complications are implant-related ones, due to instrumentation being unsupported by strong fusion, and postoperative morbidity due to repeated surgeries. In the past few years, as long-term follow-up data have become available, other, somewhat unexpected complications have turned up as well.

Complication rates in with the original, single-rod technique were significantly higher. In the series of Mineiro and Weinstein [8] published in 2002, a retrospective radiographical review of 11 patients undergoing subcutaneous rod insertion for early-onset deformity unresponsive to conservative treatment, patients were operated on using the method described by Moe et al. using Moe rods in nine and Harrington rods in two and followed for 5.1 years. The authors reported 17 complications, an average of 1.5 per patient, on a total of 53 operative procedures, index and distraction procedures combined. Rod failure was the most commonly encountered complication in this paper, occurring ten times in eight patients. Hook dislodgement in two and infection in two (one deep, one superficial) were also reported. The authors also noted clinically increased vertebral rotation despite adequate control of coronal plane deformity and concluded that the limited growth obtained during treatment did not justify its preference over fusion at an early age.

In the 2002 paper by Acaroglu et al. [9, 12] patients undergoing fusionless instrumentation without fusion were 
evaluated. The average number of lengthening operations per patient was reported to be 4.6 and the overall number of surgeries, 7. Six patients in this series developed complications, including dislodgment of hooks, rod breakage, facet or laminar fractures and surgical site infection. The patients were hospitalized for an average of 101 days during the course of the treatment. The authors also observed increased rigidity following distraction treatment at definitive surgery, in some instances requiring the use of extra interventions to increase spinal flexibility.

Complications were reported also in the study performed by Akbarnia et al. [10] and published in 2005. The authors revised the technique of subcutaneous rodding and used two rods instead of one and dedicated connectors for distraction procedures with routine lengthening without waiting for deformity to increase. They reported complications in 11 of the 23 patients in the series, but noted that most complications could be addressed during planned surgical procedures. Two patients had deep wound infections requiring surgical debridement, while four had superficial surgical site infections, and had to be addressed with unplanned surgeries. Implant-related complications consisted of two broken rods, two dislodged hooks and one screw pull-out in five patients and all could be addressed during planned lengthening surgery. The authors also reported alignment problems, with the crankshaft phenomenon occurring in one patient and one junctional kyphosis requiring extension of the implant construct. The rate for unplanned surgeries was only $4 \%$ of total surgical interventions. The authors concluded that while still reasonably high, the complication rate was not prohibitive of the technique when its multiple advantages are considered.

In another report by Akbarnia et al. [12], 13 patients followed for 3-11 years after final fusion were analyzed. Six patients (46\%) experienced complications, including the treatment period and follow-up after final fusion. Only three implant-related complications were observed during the treatment period, two of which were rod breakages that did not require unplanned surgery and were addressed during final fusion. One patient had proximal hook pullout, which required unplanned surgery. This patient also developed deep wound infection and underwent two unplanned surgical operations. Although the complication rates were still found to be high, the authors concluded that the technique was a reasonable choice in the treatment of early-onset deformity.

In a more recent report, 140 patients regardless of diagnosis were analyzed especially for complications of growing rod treatment [21]. The patients underwent 823 planned (140 index, 633 lengthenings and 50 final fusions) and 74 unplanned surgeries, with a mean number of lengthenings of 4.3 per patient and a mean interval between them of 10.4 months. The treatment was aborted in only one patient due to poor bone quality. The authors reported a total of 177 complications in 81 patients $(58 \%)$, with a mean number of complications of 2.2, $103(58 \%)$ of which could be addressed during planned surgery. Patients treated with a single rod had significantly higher complications than patients with a dual rod construct while curve correction was also found to be more favorable in the dual rod group. Double rods and subcutaneous placement of the instrumentation were found to be associated with higher woundsite problems. The authors noted that due to the protracted treatment period of early-onset deformity regardless of chosen modality, a high rate of complications are expected and can be reduced by delaying index surgery as long as possible (13\% decrease of complications for each year increase at initial surgery), using dual rods instead of a single rod and decreasing the number of lengthenings as the complication risk was found to increase by $24 \%$ for each additional surgical procedure performed.

Risk factors for rod fracture, a common complication during growing rod treatment, were analyzed in a 2011 report by Yang et al. [22]. The records of 327 patients were analyzed and 86 rod fractures observed in 49 patients, 16 of whom had repeat fractures and 8 having more than 2 . The most common breakage location was determined to be above and below the tandem connectors and near the thoracolumbar junction. Ambulatory patients, patients with syndromic scoliosis and single rods had more frequent rod breakages. The authors concluded that risk factors for rod fractures include prior breakage, single rods used, stainless steel rods over titanium rods, rods with smaller diameters, shorter tandem connectors and ambulation. Length of instrumentation, anchor type and pelvic fixation were not found to have a significant effect. Other studies to decrease implant-related complications have been published as well [23].

Another complication deserving specific mention is junctional kyphosis. When spinal motion is eliminated by fusion and forces exerted upon the spine are altered due to the presence of implants, creating significant difference of load between segments, it is impossible to avoid increased stresses at junctional regions. This localized concentration of stress often causes adjacent segment degeneration and/or the development of new deformities. Soft tissue preservation during surgery, the localization of the junctional region, the patient's bone quality, the preoperative severity of deformity and how well the sagittal contours of the spine have been restored postoperatively are all factors that influence the frequency and severity of adjacent segment problems. This complication has been defined in the pediatric age group after treatment of AIS and Scheuermann's kyphosis and how frequently it occurs after growing rod surgery is a matter of debate. Repetitive distractions during growing rod treatment may pose a risk 
factor not found in instrumentation and fusion. On the other hand, the lack of fusion in growing rod treatment may allow the spinal implant to be less rigid and therefore decrease the stresses that occur at junctional areas. This speculation has been confirmed by the experimental study performed by Yilgor et al. [24] where the authors observed that the quantity of forces exerted upon adjacent segments after growing rod application are in fact close to normal values. Bess et al. [21] have reported 3 patients with junctional kyphosis in a series of 177. Akbarnia et al. [25] have suggested proper contouring of the rod with regards to kyphosis and the careful preservation of ligaments during surgery to avoid this complication. In a recent study, Skaggs et al. [26] have proposed that this complication is actually more frequent than has been reported and that this low incidence is due to an error in measurement. However, this observation is limited to the patients of one center. It is unknown what the rate of this complication would be in series where its incidence was found to be low if the patients' data were to be re-measured accordingly to the authors' method.

Neurologic complications in straightforward growing rod surgery are rare. They may be caused by excessive distraction or significant deformity correction. Sankar et al. [27] studied the neurologic risk in growing rod surgery and questioned the need for neuromonitoring. They reviewed data from 782 growing rod surgeries, 252 of which were index surgery, 168 implant exchanges and 362 were lengthenings, $73 \%$ of which were performed with neuromonitoring. Only one injury occurred in the series, during an implant exchange (pedicle screw placement), resulting in an injury rate of $0.1 \%$. Neuromonitoring changes occurred in $0.9 \%$ of index surgeries, $0.9 \%$ of implant exchanges, and $0.5 \%$ of lengthenings. The only case where this change occurred during lengthening was a complicated case that had had a change during index surgery as well. The authors concluded that the use of neuromonitoring is justified when implants are placed for the first time or exchanged, but lengthenings, especially in patients who have had no problems during index surgery, do not seem to require routine neuromonitoring. The authors also note that their evidence is anecdotal and urge caution while interpreting their results.

The finding that increased number of surgeries causes an increased number of complications has ignited a flurry of research into the development of remotely controlled devices where lengthening can be performed without an invasive procedure. Experimental studies of these implants are scant. Takaso et al. [28] reported good results with their remote-controlled growing rod instrumentation on beagle dogs with induced scoliotic deformities. More recently, Akbarnia et al. [29] published the results of their experimental study on immature pigs instrumented with a newly designed, magnetically controlled growing rod. Five animals in the experimental growth underwent weekly spine distraction, achieving an average of $39 \mathrm{~mm}$ after a 7-week period and were compared to a sham group of three animals. While the experimental group achieved $80 \%$ of predicted spinal height with the distraction equipment, an accelerated increase in vertebral body height was observed after the magnetic implants were removed. No complications related to the implant occurred in this study.

\section{Controversies}

While there is some agreement in practice and principle regarding the use of growing rods for curves over $60^{\circ}$ in patients under the age of ten, no consensus yet exists regarding the optimal age for index surgery, the management of sagittal plane deformity, suitable diagnoses for growing rod treatment, the interval between lengthenings, types of foundations or the placement of rods subcutaneously or submuscularly [30, 31].

Although the effectiveness and reliability of the growing rod in early-onset scoliosis has been proven numerous times in the past years by reports published in prestigious journals, the negative psychological and social effects of young children having to go through multiple surgical procedures so early in their development on them and their families has become a point of discussion. In an attempt to quantify the social consequences of the growing rod, the data of 265 patients from 16 international centers were studied [32]. More than $90 \%$ of the patients undergoing growing surgery were found to be $<10$ years of age, and active treatment to take on average 5 years, which meant patients had the potential to undergo up to 12 procedures during this time frame. Five of the 16 centers experienced resistance toward regular lengthening on part of the family. This study underlines the necessity of having mutual understanding and sound cooperation with the family.

In order to analyze these issues and determine the severity of it, quality of life studies with validated outcome measures are required. A questionnaire specific to earlyonset scoliosis is now available [33]. The application of this questionnaire in large patient series with a diversity of diagnoses that are from different cultures and having these results compared with healthy subjects and patients requiring hospitalizations or multiple surgeries for pathologies other than spinal deformity will guide the way to a better understanding of the impact of treatment. It is not sufficient to measure the success of treatment only with the numbers of deformity analysis, trunk growth and pulmonary functions; quality of life needs to be assessed, and only then it is possible to speak of success or failure. 
What implant is the most favorable one for foundation/ anchor sites?

There is variation in surgeons' choice of anchor implants and no clear consensus on which implant to use. Mahar et al. [13] reported on the biomechanical comparison of various anchors and observed that screws at both anchor sites with cross-link constructs demonstrated the greatest load to failure, and all screw-only constructs were superior to constructs including hooks. These findings are concurrent with other reports regarding pedicle screw stability. Pedicle screws provide sound three-column fixation with greater pull-out strengths, making them the author's first choice for anchor fixation.

Skaggs et al. compared complications between hooks and pedicle screws and found that out of 896 pedicle screws, there were $22(2.4 \%)$ complications directly related to the screw while of 867 hooks studied, there were 60 (6.9\%) complications observed. None of the complications were associated with a neurologic or vascular injury. The authors concluded that pedicle screws in growing rods have significantly less complications than hooks [34].

Pelvic fixation is often used in growing rods applied to patients with long sweeping curves as encountered in neuromuscular and syndromic scoliosis. Sponseller et al. studied the outcomes and complications in this subgroup of by reviewing 36 patients with growing rods anchored in the pelvis and compared rod breakage rates with patients whose constructs were not fixed in the pelvis [27]. It was observed that iliac screws achieved better deformity and pelvic obliquity correction than sacral fixation. The same was found to be true for double rods as well. Rod breakage rate was not found to be significantly higher in patients with pelvic fixation than in patients without pelvic fixation. Iliac screw breakage was found to be a complication. The authors concluded that both iliac screws and rods provide satisfactory distal fixation in growing rod constructs that need to span the pelvis and that lumbar lordosis may be better preserved with these rather than spine-to-spine constructs as iliac anchors extend anterior to the center of mass. The more frequent breakages observed in iliac screws do not seem to affect outcome.

\section{How many segments should be included in the construct?}

As the growing rod technique still primarily employs distractive forces for correction, Harrington principles come into consideration when selecting anchor sites. Vertebrae within the stable zone as defined by Harrington should be used. Determination of the stable vertebrae is, in the author's experience, best performed on traction X-rays obtained under general anesthesia just before the commencement of surgery. Thoracic kyphosis should be taken into account when proximal anchor sites are chosen and rods should be contoured accordingly to avoid junctional kyphosis. Generally, this places anchor sites in high thoracic (T2-3) and high lumbar (L2-3) in idiopathic and idiopathic-like deformities. Congenital scoliosis poses a challenge for implant site selection and these patients should be considered on a case-to-case basis.

\section{Should the rods be placed in a submuscular or subcutaneous location?}

Although the technique was initially defined as the "subcutaneous rod', wound complications and implant prominence have caused surgeons to search for an alternative placement of rods. Submuscular placement offers the best alternative in this group of patients, who, due to their conditions and deformity, are generally of small stature with very little subcutaneous fat. In the 2011 paper by Bess et al. [21], it was found that subcutaneous placement in double rod constructs was associated with a significantly higher incidence of wound-site complications, while submuscular placement decreased them.

\section{What should be the frequency of lengthening?}

The first reported indication for lengthening procedures was determined to be a $10^{\circ}$ increase in deformity during follow-up after index surgery. This evolved to routine lengthening to catch up and keep pace with growth, with variable intervals of time due to reports detailing nearnormal growth with such practice $[10,12]$. It has also been observed that more frequent lengthenings result in a greater amount of growth achieved during the course of treatment [12]. However, an increased number of surgeries has been found to be associated with an increased rate of complications as well. The current trend is to perform routine lengthening every 4 months in very small children, every 6 months in most children and every 9 months when the involved segment of the spine is short [25], with complications occurring during the course of treatment addressed during planned surgery whenever possible.

\section{Does the underlying diagnosis present an indication or contraindication for growing rod treatment? Is the growing rod treatment suitable for idiopathic and idiopathic-like deformities only, or can it be used in other diagnoses as well?}

While the growing rod was initially defined for idiopathic and idiopathic-like deformities, early-onset deformity 
includes many diagnoses, including syndromic cases, cerebral palsy, congenital deformities, Marfan syndrome, SMA and others. The treatment of these incurs additional challenges, especially when their generally unresponsive nature to conservative treatment is considered.

In the 2011 study by Elsebai et al., the data of 19 patients with congenital deformities undergoing growing rod treatment were analyzed. Correction of deformity and maintenance of this correction and growth obtained during treatment were found to be comparable to results in the idiopathic-like group, with improvements in space available for the lung ratio. The complication rate was not found to be different. The authors concluded that the growing rod is a safe and effective treatment for selected patients with congenital spinal deformities.

Growing rod treatment has also been used in SMA, a disorder associated with early-onset, highly progressive curves usually unresponsive to conservative treatment and complicated with comorbidities of other systems, such as frequent pulmonary infections and poor nutritional status. McElroy et al. [18] compared 15 SMA patients with 80 juvenile/infantile idiopathic scoliosis patients undergoing growing rod treatment (Figs. 3, 4). Curve correction, pelvic obliquity and space available for the lung ratios in SMA patients improved, but the treatment was not found to halt rib cage collapse. Hospital stays in SMA patients were longer compared with patients with idiopathic deformities, but the rate of major complications was found to be lower. Chandran et al. [19] studied 11 patients with SMA types I and II undergoing growing rod treatment and observed good curve correction and a low rate of complications (only postoperative medical complications). Growing rods seem to be a viable treatment option in early-onset scoliosis associated with SMA.

Growing rod treatment in deformities associated with Marfan syndrome has also been studied. Sponseller et al. in their study included ten patients with deformities that developed before the age of 3 years, who were operated on using the growing rod technique and lengthenings performed once a year for patients on warfarin treatment. Mean curve correction for dual rods (7 of the patients) was reported to be $60 \%$, with an overall length of $11.5 \mathrm{~cm}$ obtained during the course of the study. Two rod breakages, one anchor dislodgment, and three intraoperative dural leaks were reported. The authors concluded that growing rods are an effective treatment for early-onset deformity in Marfan syndrome, helping prevent large infantile curves from becoming severe and allowing definitive fusion closer to skeletal maturity. Acute heart failure following growing rod surgery for Marfan syndrome-related scoliosis has been reported, thought to result from mechanical torsion of coronary arteries due to overdistraction. Release of distraction reversed the symptoms in this patient [35].
In a recent study by Yang et al. [36], the results of 16 patients with spinal deformity associated with cerebral palsy that were treated with growing rods were analyzed. The patients' mean deformity decreased from $83^{\circ}$ to $51^{\circ}$ and their T1-S1 length increased an average of $9 \mathrm{~cm}$ during treatment with lengthenings every 9 months. Only one significant implant-related complication was observed while there were six deep wound infections and four pneumonias. The authors' results indicate that growing rod treatment can be successfully employed in the treatment of severe scoliotic curves due to cerebral palsy.

\section{Do we reach our intended result with growing rod treatment?}

The treatment of early-onset scoliosis is a special kind of challenge. It involves a child, often too young to completely understand the intricacies of his or her treatment, and a family that is worried and anxious. Conservative treatment in itself is a long-term commitment, with restrictive and uncomfortable braces or casts worn over long periods of the child's life, often interfering with daily activities, causing its own set of complications that range from skin problems to an inability to control deformity. Its effectiveness also leaves a lot to be desired.

Considering the growing rod as what it is - a sort of temporary internal brace intended to tide the child over to as close to skeletal maturity as possible before definitive fusion is performed, and to let him or her grow in the meanwhile as well-it can be said with some confidence that it does reach its intended goal. Its complications are frequent and have many facets, from objective ones such as implant failures and wound site infections, to the psychological and familial effects of having a child forced to undergo surgery, be hospitalized and receive general anesthesia routinely every 6 months. However, it should never be forgotten that these children already struck out on the chance of a normal life with their highly progressive, severe curves, and the growing rod provides advantages that would be impossible to achieve with early fusion.

\section{Can the spine be lengthened at the same pace throughout the duration of treatment?}

It has been observed before during the course of growing rod treatment that the spine stiffens with repeated distraction procedures, in some cases going on to ankylosis of uninstrumented (and unexposed) segments. In most patients, repeated distractions increasingly require more force and achieve less length. This phenomenon has been studied. 

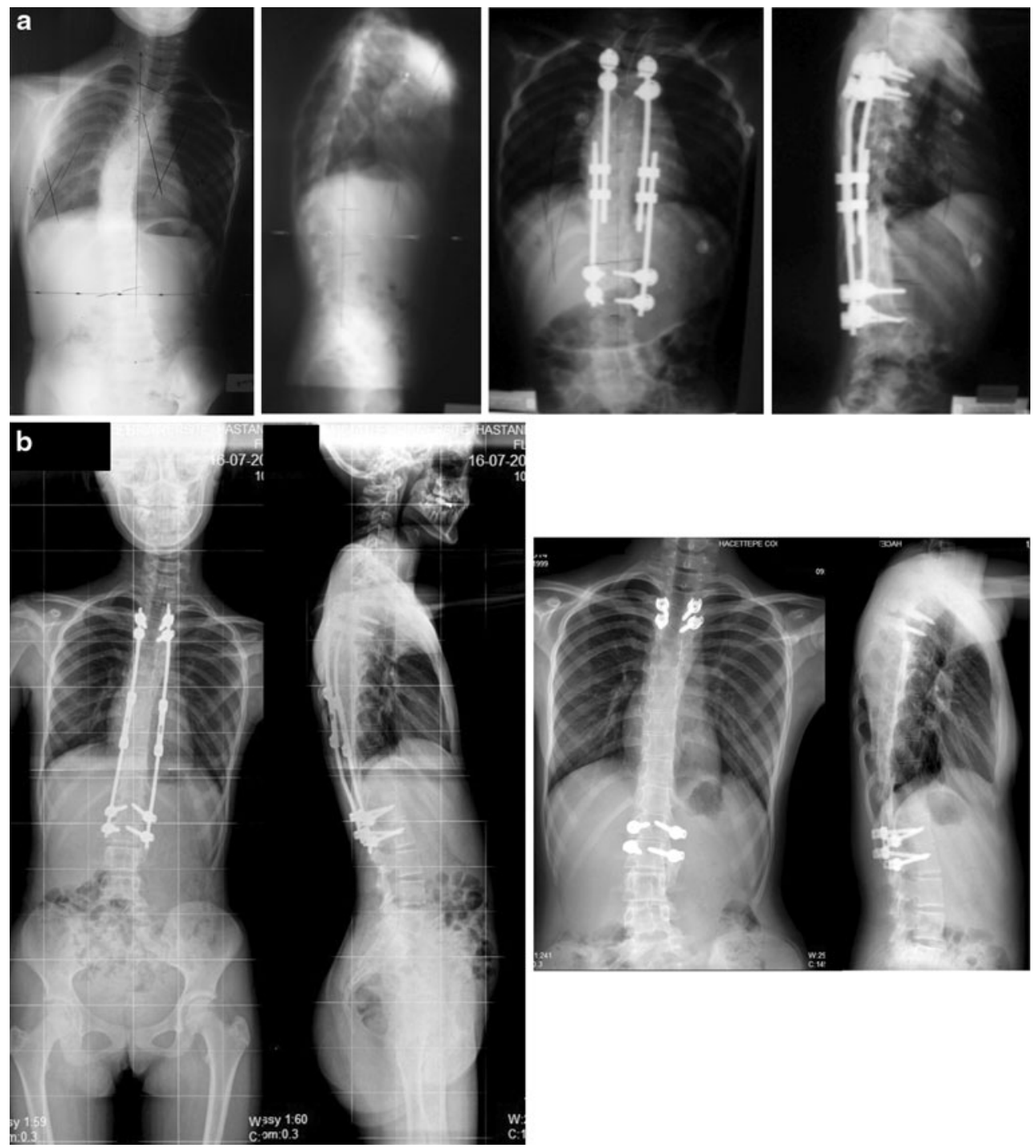

Fig. 3 The patient in this figure is a 74-month-old girl with infantile idiopathic scoliosis whose standing height at the commencement of growing rod treatment was $115 \mathrm{~cm}$. a Preoperative and immediately postoperative standing $\mathrm{X}$-rays after index surgery. The patient underwent a total of ten lengthenings and required no unplanned surgery during the treatment period. Her standing height at the conclusion of growing rod treatment was $151.5 \mathrm{~cm}$, her forced vital

Sankar et al. [37] reported on 38 patients with at least 3 lengthenings and a minimum of 2 years of follow-up and measured T1-S1 lengths after index surgery and each lengthening. They observed that major curve correction occurred after index surgery $\left(74^{\circ}\right.$ to $\left.36^{\circ}\right)$ and the average annual length gain in the T1-S1 segment was $1.76 \mathrm{~cm} /$ year. The average $\mathrm{T} 1-\mathrm{S} 1$ gain, however, decreased significantly with repeated lengthenings and when time was considered as a factor, this gain appeared to decrease over time as well. The authors concluded that their findings might be due to autofusion of the spine or immobilization by a rigid device. However, the continued gain in the capacity $2 \mathrm{~L}$ ( $76 \%$ of expected) and forced expiratory volume in the first second $94 \%$ of expected. b Standing anterior-posterior and lateral X-rays 6 months after the last lengthening. Excellent correction of deformity and establishment of trunk balance led to the decision of implant removal and observation at the conclusion of growing rod treatment. Immediate postoperative X-rays show good maintenance of correction and balance

T1-S1 distance indicates that despite possible fusion, a biologically active tissue remains that can still be stimulated to grow.

Noordeen et al. [38] measured the forces and the amount of distraction required over time in patients treated with the growing rod. They prospectively measured the distractive forces required for 60 lengthenings in 26 patients with a single submuscular rod and observed that the force required to distract the spine doubled at the fifth lengthening, and it was significantly higher than that required for the fourth lengthening. The mean length that was achieved also decreased gradually and became $8 \mathrm{~mm}$ or less by the 

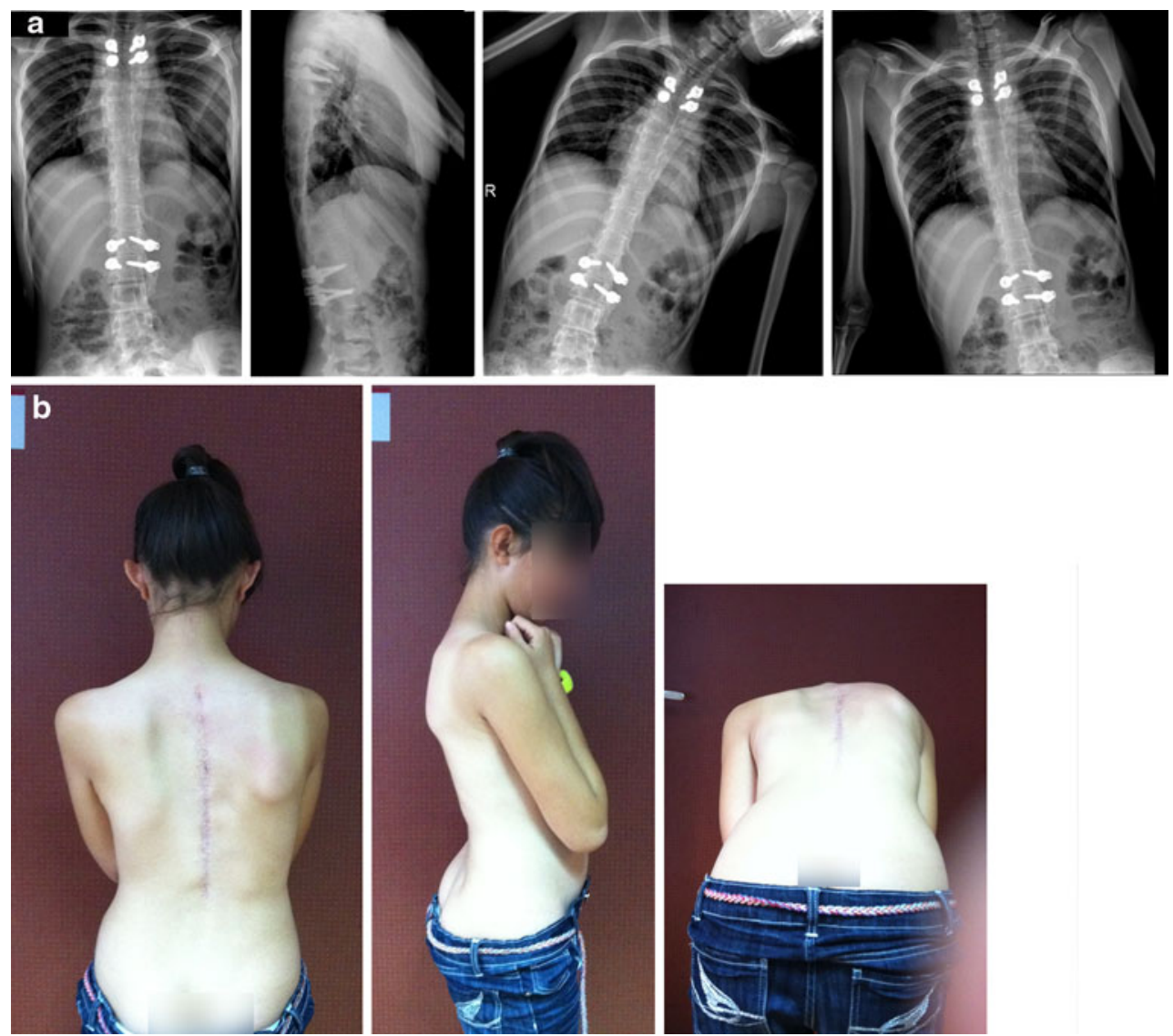

Fig. 4 a The patient from Fig. 3 returned for a follow-up visit 6 months after implant removal. Standing anterior-posterior and lateral X-rays show no increase in deformity and good coronal and sagittal alignment. However, bending X-rays clearly demonstrate that the spine is rigid and immobile despite no formal fusion (or exposure) has been performed on intermediate segments. b Patient's clinical appearance at 6 months after implant removal fifth lengthening. It can be concluded, based on this and the aforementioned study, that after five or six lengthenings, more force is needed to distract the spine and even so, less gain in height is achieved.

\section{Are there unexpected situations, and if yes, how significant are they?}

Cahill et al. [39] reported on nine patients who had undergone growing rod treatment and definitive fusion with a mean of 9.6 years of follow-up. They observed autofusion in uninstrumented segments in $89 \%$ of children and had to perform on average 7 Smith-Petersen osteotomies to achieve a Cobb angle correction of $44 \%$ at definitive surgery. The total correction during treatment was $61 \%$, while T1-S1 length increase was $11.2 \mathrm{~cm}$. The authors questioned the fusionless and growth-sparing nature of the technique but could not otherwise explain the growth achieved during treatment. It is possible that this reported fusion takes place late during the course of the treatment, and even if not, it has been shown in the past years that even unsegmented bars show growth when placed under distraction. There is no doubt that the growing rod causes the spine to stiffen and lose mobility, but there is also no doubt that growth is preserved. How does the spine continue growing if it is fused? If growth continues, how can we say there is fusion? Is one of these observations wrong? Quite possibly, they are both right. Cahill et al. noted fusion at the last stage, just before definitive fusion. Even if there is fusion in the spine, it may have occurred very late in the duration of treatment, until which time spinal growth is almost complete. Even if spontaneous fusion does take place, this fusion mass is probably a thin, weak layer of new bone formation that fractures during every lengthening with distraction. It is also conceivable that this new bone formation is a biologically active type of tissue and continues growing under distractive forces, much like in the example of the unsegmented bar that grows when it is distracted. 


\section{Do we preserve growth or stimulate it?}

Growth preservation can be achieved with routine distraction. While this determination is an important milestone in the treatment of early-onset scoliosis, it also brings with it the question of whether growth is preserved or stimulated. Patients in Akbarnia et al.'s [10, 12] series who were, for various reasons, lengthened more frequently than 6 months showed a greater amount of growth. Yilmaz et al. [40] showed in their experimental study on 12 immature pigs that vertebral growth continues during growing rod treatment at an accelerated but statistically not significant rate.

In a 2012 study performed at the authors' institution [41], vertebrae within and outside instrumentation levels in 20 patients with idiopathic-like deformities undergoing growing rod treatment were measured and compared for growth. Growth taking place in vertebrae within instrumentation levels was $7.0 \mathrm{~mm}$ compared with $5.2 \mathrm{~mm}$ in vertebrae outside instrumentation levels. Considering that the vertebrae outside instrumentation levels are lumbar and expected to grow even faster than their within-instrumentation counterparts, this study indicates that growth may be stimulated rather than preserved with growing rod treatment.

If the knowledge that complications will occur is considered and accepted, it is possible to control deformity and preserve near-normal growth with the growing rod treatment without causing excessive morbidity on patients. Even though it is by definition a fusionless technique, the vertebral column is perturbed many times during the course of treatment, and while it keeps growing, it is impossible to say that the spine is completely unaffected. This autofusion may make it possible in selected cases to remove the implants and leave the spine be without additional fusion and instrumentation procedures.

\section{Conclusion}

A young child with an early-onset deformity requires a long-term method to control his or her deformity until spinal fusion can be performed with the most minimal of disadvantages possible. The growing rod method brings this child to near skeletal maturity with height similar to his or her peers, pulmonary functions above $80 \%$ of normal, but, unfortunately, not a healthy spine.

In conclusion, the spinal surgeon is no longer forced to choose between a crooked-but-long or straight-but-short spine. We get a long, straight spine, which also happens to be rigid. No treatment at the moment can completely erase the effects of an inherent error in the spine, curing earlyonset deformity completely, but as alternatives are scant, the growing rod treatment remains our best option in the treatment of early-onset deformity regardless of etiology.
Conflict of interest None.

\section{References}

1. Dimeglio A (2001) Growth in pediatric orthopaedics. J Pediatr Orthop 21(4):549-555

2. Campbell RM Jr, Smith MD, Mayes TC et al (2003) The characteristics of thoracic insufficiency syndrome associated with fused ribs and congenital scoliosis. J Bone Joint Surg Am 85-A(3):399-408

3. Goldberg CJ, Gillic I, Connaughton O et al (2003) Respiratory function and cosmesis at maturity in infantile-onset scoliosis. Spine 28(20):2397-2406

4. Johnston CE (2011) Delaying tactics: traction, casting and bracing. In: Yazici M, EPOS (eds) Non-idiopathic spine deformities in young children, chap 9. Springer, Heidelberg, pp 109-121

5. Gillespie R, O'Brien J (1981) Harrington instrumentation without fusion. J Bone Joint Surg Br 63:461

6. Moe JH, Kharrat K, Winter RB, Cummine JL (1984) Harrington instrumentation without fusion plus external orthotic support for the treatment of difficult curvature problems in young children. Clin Orthop Relat Res 185:35-45

7. Rinsky LA, Gamble JG, Bleck EE (1985) Segmental instrumentation without fusion in children with progressive scoliosis. J Pediatr Orthop 5(6):687-690

8. Mineiro J, Weinstein SL (2002) Subcutaneous rodding for progressive spinal curvatures: early results. J Pediatr Orthop 22(3): 290-295

9. Acaroglu E, Yazici M, Alanay A, Surat A (2002) Three-dimensional evolution of scoliotic curve during instrumentation without fusion in young children. J Pediatr Orthop 22(4):492-496

10. Akbarnia BA, Marks DS, Boachie-Adjei O, Thompson AG, Asher MA (2005) Dual growing rod technique for the treatment of progressive early-onset scoliosis: a multicenter study. Spine (Phila Pa 1976) 30(17 Suppl):S46-S57

11. Thompson GH, Akbarnia BA, Kostial P et al (2005) Comparison of single and dual growing rod techniques followed through definitive surgery: a preliminary study. Spine (Phila Pa 1976) 30(18):2039-2044

12. Akbarnia BA, Breakwell LM, Marks DS et al (2008) Dual growing rod technique followed for three to eleven years until final fusion: the effect of frequency of lengthening. Spine (Phila Pa 1976) 33(9):984-990

13. Mahar AT, Bagheri R, Oka R, Kostial P, Akbarnia BA (2008) Biomechanical comparison of different anchors (foundations) for the pediatric dual growing rod technique. Spine J 8(6):933-939

14. Caubet JF, Emans JB (2011) Halo-gravity traction versus surgical release before implantation of expandable spinal devices: a comparison of results and complications in early-onset spinal deformity. J Spinal Disord Tech 24(2):99-104

15. Farooq N, Garrido E, Altaf F et al (2010) Minimizing complications with single submuscular growing rods: a review of technique and results on 88 patients with minimum two-year follow-up. Spine (Phila Pa 1976) 35(25):2252-2258

16. Schroerlucke SR, Akbarnia BA, Pawelek JB et al (2011) How does thoracic kyphosis affect patient outcomes in growing rod surgery? Spine (Phila Pa 1976) (Epub ahead of print)

17. Elsebai HB, Yazici M, Thompson GH et al (2011) Safety and efficacy of growing rod technique for pediatric congenital spinal deformities. J Pediatr Orthop 31(1):1-5

18. McElroy MJ, Shaner AC, Crawford TO et al (2011) Growing rods for scoliosis in spinal muscular atrophy: structural effects, complications, and hospital stays. Spine (Phila Pa 1976) 36(16):1305-1311 
19. Chandran S, McCarthy J, Noonan K, Mann D, Nemeth B, Guiliani T (2011) Early treatment of scoliosis with growing rods in children with severe spinal muscular atrophy: a preliminary report. J Pediatr Orthop 31(4):450-454

20. Sabourin M, Jolivet E, Miladi L, Wicart P, Rampal V, Skalli W (2010) Three-dimensional stereoradiographic modeling of rib cage before and after spinal growing rod procedures in earlyonset scoliosis. Clin Biomech (Bristol, Avon) 25(4):284-291

21. Bess S, Akbarnia BA, Thompson GH et al (2010) Complications of growing-rod treatment for early-onset scoliosis: analysis of one hundred and forty patients. J Bone Joint Surg Am 92(15): 2533-2543

22. Yang JS, Sponseller PD, Thompson GH et al (2011) Growing rod fractures: risk factors and opportunities for prevention. Spine (Phila Pa 1976) 36(20):1639-1644

23. Sankar WN, Acevedo DC, Skaggs DL (2010) Comparison of complications among growing spinal implants. Spine (Phila $\mathrm{Pa}$ 1976) 35(23):2091-2096

24. Yilgor C, Aritan S, Demirkran G, Daglioglu K, Kosemetmetoglu K, Yazici M (2011) Does growing rod cause degeneration and secondary deformity in the adjacent segment? J Child Orthop 5:394-395

25. Akbarnia BA, Emans JB (2010) Complications of growth-sparing surgery in early onset scoliosis. Spine (Phila Pa 1976) 35(25): 2193-2204

26. Skaggs DL, Myung KS, Lee C (2011) Proximal junctional kyphosis in distraction-based growing rods. Free paper, the 5th international congress on early onset scoliosis and growing spine (ICEOS), 18-19 November 2011, Orlando, Florida

27. Sankar WN, Skaggs DL, Emans JB et al (2009) Neurologic risk in growing rod spine surgery in early onset scoliosis: is neuromonitoring necessary for all cases? Spine (Phila Pa 1976) 34(18): 1952-1955

28. Takaso M, Moriya H, Kitahara H et al (1998) New remote-controlled growing-rod spinal instrumentation possibly applicable for scoliosis in young children. J Orthop Sci 3(6):336-340

29. Akbarnia BA, Mundis GM Jr, Salari P, Yaszay B, Pawelek JB (2011) Innovation in growing rod technique: a study of safety and efficacy of a magnetically controlled growing rod in a porcine model*. Spine (Phila Pa 1976) (Epub ahead of print)
30. Yang JS, McElroy MJ, Akbarnia BA et al (2010) Growing rods for spinal deformity: characterizing consensus and variation in current use. J Pediatr Orthop 30(3):264-270

31. Vitale MG, Gomez JA, Matsumoto H, Roye DP Jr (2011) Variability of expert opinion in treatment of early-onset scoliosis. Clin Orthop Relat Res 469(5):1317-1322

32. Akbarnia BA, Campbell R, Dimeglio A et al (2011) Fusionless procedures for the management of early-onset spine deformities in 2011: what do we know? J Child Orthop 5(3):159-172

33. Corona J, Matsumoto H, Roye DP, Vitale MG (2011) Measuring quality of life in children with early onset scoliosis: development and initial validation of the early onset scoliosis questionnaire. J Pediatr Orthop 31(2):180-185

34. Skaggs DL, Myung KS, Johnston CE, Akbarnia BA, Group GSS (2010) Pedicle screws have fewer complications than hooks in children with growing rods. International Congress on Early Onset Scoliosis and Growing Spine, Toronto

35. Skaggs DL, Bushman G, Grunander T, Wong PC, Sankar WN, Tolo VT (2008) Shortening of growing-rod spinal instrumentation reverses cardiac failure in child with Marfan syndrome and scoliosis. A case report. J Bone Joint Surg Am 90(12):2745-2750

36. Sponseller PD, Wild AT, Growing Spine Study Group (2010) Growing rods for cerebral palsy. J Child Orthop 4:495-496

37. Sankar WN, Skaggs DL, Yazici M et al (2011) Lengthening of dual growing rods and the law of diminishing returns. Spine (Phila Pa 1976) 36(10):806-809

38. Noordeen HM, Shah SA, Elsebaie HB, Garrido E, Farooq N, Al Mukhtar M (2011) In vivo distraction force and length measurements of growing rods: which factors influence the ability to lengthen? Spine (Phila Pa 1976) 36(26):2299-2303

39. Cahill PJ, Marvil S, Cuddihy L et al (2010) Autofusion in the immature spine treated with growing rods. Spine (Phila Pa 1976) 35(22):E1199-E1203

40. Yilmaz G, Huri G, Demirkran G et al (2010) The effect of posterior distraction on vertebral growth in immature pigs: an experimental simulation of growing rod technique. Spine (Phila Pa 1976) 35(7):730-733

41. Olgun ZD, Ahmadiadli H, Alanay A, Yazici M (2012) Vertebral body growth during growing rod instrumentation: growth preservation or stimulation? J Pediatr Orthop 32(2):184-189 